\title{
THE BOUNDARY HARNACK INEQUALITY FOR SOLUTIONS TO EQUATIONS OF ARONSSON TYPE IN THE PLANE
}

\author{
Niklas L. P. Lundström and Kaj Nyström \\ Umeå University, Department of Mathematics and Mathematical Statistics \\ S-901 87 Umeå, Sweden; niklas.lundstrom@math.umu.se \\ Umeå University, Department of Mathematics and Mathematical Statistics \\ S-901 87 Umeå, Sweden; kaj.nystrom@math.umu.se
}

\begin{abstract}
In this paper we prove a boundary Harnack inequality for positive functions which vanish continuously on a portion of the boundary of a bounded domain $\Omega \subset \mathbf{R}^{2}$ and which are solutions to a general equation of $p$-Laplace type, $1<p<\infty$. We also establish the same type of result for solutions to the Aronsson type equation $\nabla(F(x, \nabla u)) \cdot F_{\eta}(x, \nabla u)=0$. Concerning $\Omega$ we only assume that $\partial \Omega$ is a quasicircle. In particular, our results generalize the boundary Harnack inequalities in [BL] and [LN2] to operators with variable coefficients.
\end{abstract}

\section{Introduction}

Recently there have been several breakthroughs in the study of boundary Harnack inequalities for $p$-harmonic functions, $1<p \leq \infty$, which vanish on a portion of the boundary of a bounded domain $\Omega \subset \mathbf{R}^{n}$. In particular, in the plane, i.e., $\Omega \subset \mathbf{R}^{2}$, the boundary Harnack inequality for $p$-harmonic functions, $1<p<\infty$, was proved in [BL] assuming only that $\Omega \subset \mathbf{R}^{2}$ is bounded and that $\partial \Omega$ is a quasicircle. In [LN2] this result was extended to $p=\infty$ and hence to the case of infinity harmonic functions. Furthermore, in [LN1] and [LN4] a number of results concerning the boundary behavior of positive $p$-harmonic functions, $1<p<\infty$, in a bounded Lipschitz domain $\Omega \subset \mathbf{R}^{n}$ were proved. In particular, the boundary Harnack inequality as well as Hölder continuity for ratios of positive $p$-harmonic functions, $1<p<\infty$, vanishing on a portion of $\partial \Omega$ were established. Furthermore, the $p$-Martin boundary problem at $w \in \partial \Omega$ was resolved under the assumption that $\Omega$ is either convex, $C^{1}$ regular or a Lipschitz domain with small constant. In [LN3] the boundary Harnack inequality and Hölder continuity for ratios of $p$-harmonic functions vanishing on a portion of certain Reifenberg flat and Ahlfors regular NTA-domains were established and the $p$-Martin boundary problem was resolved in these domains. Finally, in the case of certain Reifenberg flat domains, these results where generalized to equations of $p$-laplace type in [LLuN], and to equations of $p$-laplace with lower order terms in [ALuN].

The purpose of this paper is to generalize the result on boundary Harnack inequalities proved in [BL] and [LN2] to more general operators of $p$-Laplace type and to operators of Aronsson type. The latter operators represent generalizations of the

doi:10.5186/aasfm.2011.3616

2010 Mathematics Subject Classification: Primary 35J25, 35J70.

Key words: Boundary Harnack inequality, $p$-Laplace, $A$-harmonic function, infinity harmonic function, Aronsson type equation, quasicircle. 
so called infinity-Laplacian. In particular, we generalize the results in [BL] and [LN2] to operators with variable coefficients.

To state our main result we need to introduce some notation. In particular, we let $\bar{E}, \partial E$, diam $E$, be the closure, boundary, diameter, of the set $E \subset \mathbf{R}^{2}$. We define $d(y, E)$ to equal the distance from $y \in \mathbf{R}^{2}$ to $E,\langle\cdot, \cdot\rangle$ denotes the standard inner product on $\mathbf{R}^{2}$ and we let $|x|=\langle x, x\rangle^{1 / 2}$ be the Euclidean norm of $x . B(x, r)=\{y \in$ $\left.\mathbf{R}^{2}:|x-y|<r\right\}$ is defined whenever $x \in \mathbf{R}^{2}, r>0$, and we let $d x$ denote the two dimensional Lebesgue measure on $\mathbf{R}^{2}$. If $O \subset \mathbf{R}^{2}$ is open and $1 \leq q \leq \infty$, then by $W^{1, q}(O)$ we denote the Sobolev space, i.e., the space of equivalence classes of functions $f$ with weak gradient $\nabla f=\left(f_{x_{1}}, \ldots, f_{x_{n}}\right)$, both of which are $q$-th power integrable on $O$. Let $\|f\|_{1, q}=\|f\|_{q}+\||\nabla f|\|_{q}$ be the norm in $W^{1, q}(O)$ where $\|\cdot\|_{q}$ denotes the usual Lebesgue $q$ norm in $O$. Note that if $q=\infty$, then $\|f\|_{1, \infty}=\|f\|_{\infty}+\|\mid \nabla f\|_{\infty}$ where $\|\cdot\|_{\infty}$ denotes the essential supremum on $O$. Next let $C_{0}^{\infty}(O)$ be the set of infinitely differentiable functions with compact support in $O$ and let $W_{0}^{1, q}(O)$ be the closure of $C_{0}^{\infty}(O)$ in the norm of $W^{1, q}(O)$. By $C(O)$ and $C_{0}(O)$ we denote the set of continuous functions on $O$ and the set of continuous functions with compact support in $O$ respectively. By $\nabla \cdot$ we denote the divergence operator.

We now introduce the geometric notions used in this paper. To start with we recall that a Jordan curve $J$ is said to be a $k$ quasicircle, $0<k<1$, if $J=h(\partial B(0,1))$ where $h \in W^{1,2}\left(\mathbf{R}^{2}\right)$ is a homeomorphism of $\mathbf{R}^{2}$ and

$$
\left|h_{\bar{z}}\right| \leq k\left|h_{z}\right|, d x \text { almost everywhere in } \mathbf{R}^{2} .
$$

Here we are using complex notation, $i=\sqrt{-1}, z=x_{1}+i x_{2}, 2 h_{\bar{z}}=h_{x_{1}}+i h_{x_{2}}$, $2 h_{z}=h_{x_{1}}-i h_{x_{2}}$. We say that $J$ is a quasicircle if $J$ is a $k$ quasicircle for some $0<k<1$. Let $w_{1}, w_{2}$ be distinct points on the Jordan curve $J$ and let $J_{1}, J_{2}$ be the arcs on $J$ with endpoints $w_{1}, w_{2}$. Then $J$ is said to satisfy the Ahlfors three point condition provided there exists $1 \leq \widetilde{M}<\infty$ such that

$$
\min \left\{\operatorname{diam} J_{1}, \operatorname{diam} J_{2}\right\} \leq \widetilde{M}\left|w_{1}-w_{2}\right|
$$

whenever $w_{1}, w_{2} \in J . \Omega$ is said to be a uniform domain provided there exists $\widehat{M}$, $1 \leq \widehat{M}<\infty$, such that if $w_{1}, w_{2} \in \Omega$, then there exists a rectifiable curve $\gamma:[0,1] \rightarrow \Omega$ with $\gamma(0)=w_{1}, \gamma(1)=w_{2}$, such that if $H^{1}(\cdot)$ denotes the one-dimensional Hausdorff measure on $\gamma$, then

$$
\begin{array}{ll}
\text { (i) } & H^{1}(\gamma) \leq \widehat{M}\left|w_{1}-w_{2}\right| \\
\text { (ii) } & \min \left\{H^{1}(\gamma([0, t])), H^{1}(\gamma([t, 1]))\right\} \leq \widehat{M} d(\gamma(t), \partial \Omega) .
\end{array}
$$

Furthermore, a bounded domain $\Omega$ is called non-tangentially accessible (NTA) if there exist $M \geq 2$ and $r_{0}$ such that the following are fulfilled:

(i) corkscrew condition: for any $w \in \partial \Omega, 0<r<r_{0}$, there exists $a_{r}(w) \in \Omega$ satisfying $M^{-1} r<\left|a_{r}(w)-w\right|<r, d\left(a_{r}(w), \partial \Omega\right)>M^{-1} r$

(ii) $\mathbf{R}^{n} \backslash \bar{\Omega}$ satisfies the corkscrew condition,

(1.4) (iii) uniform condition: if $w \in \partial \Omega, 0<r<r_{0}$ and $w_{1}, w_{2} \in B(w, r) \cap \Omega$, then there exists a rectifiable curve $\gamma:[0,1] \rightarrow \Omega$ with $\gamma(0)=w_{1}, \gamma(1)=w_{2}$ s.t.

(a) $H^{1}(\gamma) \leq M\left|w_{1}-w_{2}\right|$

(b) $\min \left\{H^{1}(\gamma([0, t])), H^{1}(\gamma([t, 1]))\right\} \leq M d(\gamma(t), \partial \Omega)$. 
We note that (iii) in (1.4) is different but equivalent to the usual Harnack chain condition given in [JK] (see [BL, Lemma 2.5]). In the following, $M$ and $r_{0}$ will be called the NTA-constants of $\Omega$. Moreover, we note that $\Omega$ is a uniform domain if and only if (1.4) (i) and (iii) hold. Furthermore, the following conditions are equivalent for $\Omega \in \mathbf{R}^{2}$ :

(i) $\partial \Omega$ is a quasicircle,

(ii) $\partial \Omega$ is a Jordan curve and satisfies the Ahlfors three point condition,

(iii) $\partial \Omega$ is a Jordan curve and $\Omega$ is a uniform domain,

(iv) $\partial \Omega$ is a Jordan curve and $\Omega$ is an NTA-domain.

For more on these geometric notions and proofs of the stated statements we refer to [G].

We next introduce the operators of $p$-Laplace type which we consider in this paper.

Definition 1.1. Let $p \in(1, \infty)$ and let $\lambda, \Lambda \in \mathbf{R}, 0<\lambda \leq \Lambda<\infty$. Let $A=\left(A_{1}, A_{2}\right): \mathbf{R}^{2} \times \mathbf{R}^{2} \rightarrow \mathbf{R}^{2}$, assume that the mapping $x \rightarrow A(x, \eta)$ is measurable for all $\eta \in \mathbf{R}^{2}$ and that the mapping $\eta \rightarrow A(x, \eta)$ is continuous for almost every $x \in \mathbf{R}^{2}$. We say that the function $A$ belongs to the class $A_{p}(\lambda, \Lambda)$ if the following conditions are satisfied for almost every $x \in \mathbf{R}^{2}$ whenever $\xi, \zeta \in \mathbf{R}^{2}$ and $\eta \in \mathbf{R}^{2} \backslash\{0\}$ :

$$
\begin{aligned}
\text { (i) } & |A(x, \xi)| \leq \Lambda|\xi|^{p-1}, \\
\text { (ii) } & \langle A(x, \xi)-A(x, \zeta), \xi-\zeta\rangle \geq \lambda(|\xi|+|\zeta|)^{p-2}|\xi-\zeta|^{2}, \\
\text { (iii) } & A(x, \eta)=|\eta|^{p-1} A(x, \eta /|\eta|) .
\end{aligned}
$$

If $A$ belongs to the class $A_{p}(\lambda, \Lambda)$, then we write $A \in A_{p}(\lambda, \Lambda)$.

Definition 1.2. Let $p \in(1, \infty)$ be given and let $A \in A_{p}(\lambda, \Lambda)$ for some $(\lambda, \Lambda)$, $0<\lambda \leq \Lambda<\infty$. Given a bounded domain $G \subset \mathbf{R}^{2}$ we say that $u$ is $A$-harmonic in $G$ provided $u$ is a weak solution of $\nabla \cdot A(x, \nabla u)=0$ in $G$, i.e., $u \in W^{1, p}(G)$ and

$$
\int_{G}\langle A(x, \nabla u), \nabla \psi\rangle d x=0
$$

whenever $\psi \in W_{0}^{1, p}(G)$. As a short notation for (1.5) we write that $\Delta_{p, A} u=\nabla$. $A(x, \nabla u)=0$ in $G$.

We note that an important class of equations which is covered by Definition 1.1 and 1.2 is the class of equations of the type

$$
\nabla \cdot\left[\langle a(x) \nabla u, \nabla u\rangle^{p / 2-1} a(x) \nabla u\right]=0 \text { in } G
$$

where $a(x)=\left\{a_{i j}(x)\right\}$ is a matrix such that the conditions in Definition 1.1 (i) and (ii) are fulfilled.

Let $p \in(1, \infty)$ be given. We are now ready to state our first results which concern $A$-harmonic functions. Given a domain $\Omega \subset \mathbf{R}^{2}$ we in the following let $\Delta(w, r)=\partial \Omega \cap B(w, r)$ whenever $w \in \partial \Omega, 0<r$. We prove the following theorems.

Theorem 1.3. Let $\Omega \subset \mathbf{R}^{2}$, assume that $\partial \Omega$ is a Jordan curve and $\Omega$ is a uniform domain with constant $\widehat{M}$. Let $p \in(1, \infty)$ be given and assume that $A \in A_{p}(\lambda, \Lambda)$ for some $(\lambda, \Lambda)$. Let $w \in \partial \Omega, 0<r \leq r_{0}$ and suppose that $u$ and $v$ are positive 
$A$-harmonic functions in $\Omega \cap B(w, r)$, continuous on $\bar{\Omega} \cap B(w, r)$ with $u=0=v$ on $\Delta(w, r)$. Then there exists a constant $c$, depending only on $\widehat{M}, p, \Lambda / \lambda$, such that if $\tilde{r}=r / c$ and $x \in \Omega \cap B(w, \tilde{r})$, then

$$
c^{-1} \frac{u\left(a_{\tilde{r}}(w)\right)}{v\left(a_{\tilde{r}}(w)\right)} \leq \frac{u(x)}{v(x)} \leq c \frac{u\left(a_{\tilde{r}}(w)\right)}{v\left(a_{\tilde{r}}(w)\right)} .
$$

Theorem 1.4. Let $\Omega, p, A, w, r, u$ and $v$ be as in Theorem 1.3. Then there exists $\hat{p}>2$ such that the following is true whenever $\hat{p} \leq p<\infty$. There exists a constant $c$, depending only on $\widehat{M}, \hat{p}, \Lambda / \lambda$, such that if $\tilde{r}=r / c$ and $x \in \Omega \cap B(w, \tilde{r})$, then

$$
c^{-1} \frac{u\left(a_{\tilde{r}}(w)\right)}{v\left(a_{\tilde{r}}(w)\right)} \leq \frac{u(x)}{v(x)} \leq c \frac{u\left(a_{\tilde{r}}(w)\right)}{v\left(a_{\tilde{r}}(w)\right)} .
$$

Theorem 1.3 was proved in [BL] in the case of the $p$-Laplace operator, i.e., the case $A(x, \eta)=|\eta|^{p-2} \eta$ while, still in the case of the $p$-Laplace operator, Theorem 1.4 was established in [LN2]. Still, Theorem 1.3 and Theorem 1.4 are new for the general class operators of $p$-Laplace type defined by the class $A_{p}(\lambda, \Lambda)$.

We next introduce the operators of Aronsson type which we consider in this paper.

Definition 1.5. Let $\alpha, \beta, \gamma, \bar{p}, \bar{c} \in \mathbf{R}_{+}$. Let $F: \mathbf{R}^{2} \times \mathbf{R}^{2} \rightarrow \mathbf{R}$ and assume that

(i $\left.\mathrm{i}_{1}\right) \quad x \rightarrow F(x, \eta)$ is differentiable for all $\eta \in \mathbf{R}^{2}$,

$\eta \rightarrow F(x, \eta)$ is strictly convex and differentiable for all $x \in \mathbf{R}^{2}$,

$\left(\mathrm{ii}_{1}\right) \quad(x, \eta) \rightarrow \nabla_{x} F(x, \eta),(x, \eta) \rightarrow \nabla_{\eta} F(x, \eta)$ are continuous for all $(x, \eta) \in \mathbf{R}^{2} \times \mathbf{R}^{2}$,

(iii $\left.{ }_{1}\right) \quad F_{x_{j} \eta_{j}}(x, \eta)$ exists and is continuous whenever $j \in\{1,2\}$, $(x, \eta) \in \mathbf{R}^{2} \times \mathbf{R}^{2}$.

Assume also that the following hold for all $x \in \mathbf{R}^{2}, \xi, \zeta \in \mathbf{R}^{2}$ and $\eta \in \mathbf{R}^{2} \backslash\{0\}$, $p \geq \bar{p}$

$$
\begin{aligned}
& \left(\mathrm{i}_{2}\right) \quad \alpha|\xi|^{2} \leq F(x, \xi) \leq \beta|\xi|^{2} \\
& \left(\mathrm{ii}_{2}\right) \quad\left(F(x, \xi)^{\frac{p-2}{2}} \nabla_{\xi} F(x, \xi)-F(x, \zeta)^{\frac{p-2}{2}} \nabla_{\xi} F(x, \zeta)\right) \cdot(\xi-\zeta) \geq \gamma^{p}|\xi-\zeta|^{p}, \\
& \left(\mathrm{iii}_{2}\right) \quad F(x, \eta)=|\eta|^{2} F(x, \eta /|\eta|) .
\end{aligned}
$$

We say that a function $F$ belongs to the class $F(\alpha, \beta, \gamma, \bar{p}, \bar{c})$ if $F$ satisfies the conditions in $\left(\mathrm{i}_{1}\right)-\left(\mathrm{iii}_{1}\right),\left(\mathrm{i}_{2}\right)-\left(\mathrm{iii}_{2}\right)$ and if for every ball $B(w, r) \subset \mathbf{R}^{2}$, there exist constants $C>0$ and $0<\kappa \leq 1$ such that the following holds for all $\eta \in \mathbf{R}^{2}$ and for all $x, y \in B(w, r)$ :

$$
\begin{aligned}
\left(\mathrm{i}_{3}\right) & \left|\nabla_{x} F(x, \eta)\right| \leq C|\eta|^{2}, \\
\left(\mathrm{ii}_{3}\right) & \left|\nabla_{x} F(x, \eta)-\nabla_{x} F(y, \eta)\right| \leq C|\eta|^{2}|x-y|^{\kappa}, \\
\left(\mathrm{iii}_{3}\right) & \left|\nabla_{\eta} F(x, \eta)-\nabla_{\eta} F(y, \eta)\right| \leq C|\eta||x-y|^{1 / 2+\kappa} .
\end{aligned}
$$

If $F$ belongs to the class $F(\alpha, \beta, \gamma, \bar{p}, \bar{c})$, then we write $F \in F(\alpha, \beta, \gamma, \bar{p}, \bar{c})$.

Definition 1.6. Let $F \in F(\alpha, \beta, \gamma, \bar{p}, \bar{c})$ for some $(\alpha, \beta, \gamma, \bar{p}, \bar{c})$. Given a bounded domain $G \subset \mathbf{R}^{2}$ we say that $u$ is $F$-infinity harmonic in $G$ provided $u$ solves the partial 
differential equation

$$
\Delta_{\infty, F} u=\nabla(F(x, \nabla u(x))) \cdot \nabla_{\eta} F(x, \nabla u(x))=0
$$

in the viscosity sense.

The partial differential equation $\Delta_{\infty, F} u$ in Definition 1.6 is referred to as an equation of Aronsson type and we recall that this type of equations was first introduced by Aronsson in [A1-A4]. These equations play an important role as the governing equation in certain minimization problems in the $L^{\infty}$-norm. Moreover, to understand the rationale for all of the conditions stated in Definition 1.5, we note that in $[\mathrm{Ju}]$ the fundamental work in $[\mathrm{J}]$ on the infinity-Laplacian was generalized to the partial differential equation in (1.10) using the assumptions stated in Definition 1.5. In particular, in [Ju, Theorem 2.3, Corollary 3.8 and Theorem 4.25] it is proved that if $f \in W^{1, \infty}(G) \cap C(\bar{G})$, then there exists a unique viscosity solution to the Dirichlet problem

$$
\Delta_{\infty, F} u=0 \text { in } G, \quad \lim _{x \in G, x \rightarrow y} u(x)=f(y) \text { for all } y \in \partial G .
$$

Moreover, in [Ju, Corollary 4.33] it is proved that if $f: \partial G \rightarrow \mathbf{R}$ is a continuous function, then the Dirichlet problem in (1.11) has a unique viscosity solution in $G$. Note that viscosity solutions are by definition continuous and that the conclusions on the solvability of the Dirichlet problem in (1.11) are valid for any bounded domain $G \subset \mathbf{R}^{2}$. Furthermore, using the assumptions in Definition 1.5, the equation in (1.11) was derived in [Ju, Corollary 3.8] as the Euler equation for so called variational Fabsolute minimizers. In particular, an F-absolute minimizer is a function $u \in$ $W_{\text {loc }}^{1, \infty}(G)$ such that

$$
\|F(x, \nabla u(x))\|_{\infty, D} \leq\|F(x, \nabla v(x))\|_{\infty, D}
$$

whenever $D$ is open, $\bar{D}$ is a compact subset of $G$ and $v \in W^{1, \infty}(D)$ is such that $u-v \in$ $C_{0}(D)$. For more on this minimization problem and questions concerning existence and uniqueness of solutions to equations of Aronsson type, as well as applications to image processing and game theory, we refer to $[\mathrm{J}],[\mathrm{JWY}],[\mathrm{Ju}],[\mathrm{BEJ}]$ and the references in these papers.

We are now ready to state our result which concern $F$-infinity harmonic functions, $F \in F(\alpha, \beta, \gamma, \bar{p}, \bar{c})$.

Theorem 1.7. Let $\Omega \subset \mathbf{R}^{2}$, assume that $\partial \Omega$ is a Jordan curve, $\Omega$ is a uniform domain with constant $\widehat{M}$ and $F \in F(\alpha, \beta, \gamma, \bar{p}, \bar{c})$ for some $(\alpha, \beta, \gamma, \bar{p}, \bar{c})$. Let $w \in \partial \Omega$, $0<r \leq r_{0}$ and suppose that $u$ and $v$ are positive $F$-infinity harmonic functions in $\Omega \cap B(w, r)$, continuous on $\bar{\Omega} \cap B(w, r)$ with $u=0=v$ on $\Delta(w, 4 r)$. Then there exists a constant $c$, depending only on $\alpha, \beta, \gamma, \bar{p}, \bar{c}, \widehat{M}$, such that if $\tilde{r}=r / c$ and $x \in \Omega \cap B(w, \tilde{r})$, then

$$
c^{-1} \frac{u\left(a_{\tilde{r}}(w)\right)}{v\left(a_{\tilde{r}}(w)\right)} \leq \frac{u(x)}{v(x)} \leq c \frac{u\left(a_{\tilde{r}}(w)\right)}{v\left(a_{\tilde{r}}(w)\right)} .
$$

Theorem 1.7 was proved in [LN2] in the case of the infinity-Laplacian, but to our knowledge no boundary Harnack inequalities for solutions of equations of Aronsson type have previously been established and hence Theorem 1.7 is new. As mentioned above we impose the restriction $F \in F(\alpha, \beta, \gamma, \bar{p}, \bar{c})$ to be able to use framework and results in $[\mathrm{Ju}]$. Moreover, in this paper we do not discuss to what extent the quite 
lengthy list of conditions stated in Definition 1.5 are necessary for the validity of the results in $[\mathrm{Ju}]$ and for the validity of Theorem 1.7 .

Concerning proofs, the proof of Theorem 1.3, Theorem 1.4 and Theorem 1.7 follow along the lines of the corresponding proofs in the case of the $p$-Laplacian, see $[\mathrm{BL}]$, and in the case of the infinity-Laplacian, see [LN2]. Though we claim limited originality here we claim that our results stresses the generality of the technique, applicable only in $\mathbf{R}^{2}$ though, developed in [BL] for $p$-harmonic functions and then refined in [LN2] to the case of infinity harmonic functions. In particular, to understand the relation between Theorem 1.3, Theorem 1.4 and Theorem 1.7 and their proofs we let $F \in F(\alpha, \beta, \gamma, \bar{p}, \bar{c})$ for some $(\alpha, \beta, \gamma, \bar{p}, \bar{c})$ and define

$$
A(x, \eta)=F(x, \eta)^{(p-2) / 2} \nabla_{\eta} F(x, \eta) .
$$

Then $A \in A_{p}(\lambda, \Lambda)$ for some $(\lambda, \Lambda)$ which only depend on $(\alpha, \beta, \gamma, \bar{p}, \bar{c})$. Let $p \in$ $(1, \infty), G$ be a bounded domain and let $f \in W^{1, p}(\Omega) \cap C(\bar{\Omega})$. Then there exists, see Lemma 2.A below, a unique weak solution $u_{p}$ to the Dirichlet problem

$$
\begin{aligned}
\nabla \cdot A\left(x, \nabla u_{p}\right) & =\nabla \cdot\left(F\left(x, \nabla u_{p}\right)^{(p-2) / 2} \nabla_{\eta} F\left(x, \nabla u_{p}\right)\right)=0 \text { in } G, \\
\lim _{x \in G, x \rightarrow y} u_{p}(x) & =f(y) \text { for all } y \in \partial G,
\end{aligned}
$$

Moreover, to describe the relation between the problems in (1.11) and (1.15) we assume, in addition, that $f \in W^{1, \infty}(G) \cap C(\bar{G})$ and we let $u_{\infty} \in W^{1, \infty}(G) \cap C(\bar{G})$ be the unique viscosity solution to (1.11) with boundary data defined by $f$. Then, arguing as in [Ju, Theorem 1.15, Proposition 2.5 and Corollary 3.8] we see that there exists a sequence $\left\{p_{j}\right\}, p_{j} \rightarrow \infty$ as $j \rightarrow \infty$, such that $u_{p_{j}} \rightarrow u_{\infty}$ uniformly in $G$ as $j \rightarrow \infty$. In particular, the unique solution $u_{\infty}$ to the Dirichlet problem (1.11) for the operator defined in Definition 1.6 is the uniform limit, as $p_{j} \rightarrow \infty$, of the corresponding unique solutions $\left\{u_{p_{j}}\right\}$ to the problems in (1.15). This conclusion allows us to derive Theorem 1.7 from Theorem 1.4.

The rest of the paper is organized in the following way. In Section 2 we prove a number of estimates for $A$-harmonic functions and in particular we establish estimates which are uniform in $p$ for $p$ large enough. Then, in Section 3 we prove Theorem 1.31.7 .

\section{Estimates for $A$-harmonic functions}

We begin this section by introducing and recalling some notation. Let $\max _{E} u$ and $\min _{E} u$ be the essential supremum and infimum of $u$ on $E$ whenever $E \subset \mathbf{R}^{n}$ and whenever $u$ is defined on $E$. Recall that $\Delta(w, r)=\partial \Omega \cap B(w, r)$ whenever $w \in \partial \Omega$, $0<r$ and that $a_{r}(w)$ denotes an interior corkscrew point of $\Omega$ guaranteed by $(i)$ in (1.4). Throughout the paper $c$ will denote, unless otherwise stated, a constant $\geq 1$, not necessarily the same at each occurrence, which is independent of $p$ but may depend on $M$ and $\hat{p}$. In general, $c\left(a_{1}, \ldots, a_{n}\right)$ denotes a constant $\geq 1$, not necessarily the same at each occurrence, which is independent of $p$ but depends on $a_{1}, \ldots, a_{n}$.

We begin with some preliminaries concerning the solvability of the Dirichlet problem and concerning the possibility to make coordinate transformations.

Lemma 2.A. Let $\Omega \subset \mathbf{R}^{2}$, assume that $\partial \Omega$ is a Jordan curve and $\Omega$ is a uniform domain with constant $\widehat{M}$. Let $p \in(1, \infty), A \in A_{p}(\lambda, \Lambda)$ for some $(\lambda, \Lambda)$ and $f \in$ 
$W^{1, p}(\Omega) \cap C(\bar{\Omega})$. Then there exists a unique solution to the Dirichlet problem

$$
\Delta_{p, A} u=\nabla \cdot A(x, \nabla u)=0 \text { in } \Omega, \quad \lim _{x \in \Omega, x \rightarrow y} u(x)=f(y) \text { for all } y \in \partial \Omega .
$$

Proof. For a detailed proof we refer the reader to [HKM, Theorem 3.17 and Theorem 6.31]. We just note that the proof of existence is based on the theory of monotone operators while the proof of uniqueness follows from the comparison principle for $A$-harmonic functions. That $\lim _{x \rightarrow y} u(x)$ exists and equals $f(y)$ for all $y \in \partial \Omega$ follows from the fact that all points on $\partial \Omega$ are $A$-regular. In particular, by (ii) in (1.4) we see that $\mathbf{R}^{2} \backslash \Omega$ has a corkscrew at each point on $\partial \Omega$. Hence, using [HKM, Theorem 6.31] we can conclude that $\mathbf{R}^{2} \backslash \Omega$ is $(p, \mu)$-thick at each point on $\partial \Omega$. As a consequence, see [HKM, Corollary 6.28], all points on $\partial \Omega$ are $A$-regular. This completes the proof.

Lemma 2.B. Let $\Omega \subset \mathbf{R}^{2}, p \in(1, \infty), A \in A_{p}(\lambda, \Lambda)$ for some $(\lambda, \Lambda)$ and assume that $u$ is an $A$-harmonic function in $\Omega$. Let $k \in \mathbf{R}$ and $z \in \mathbf{R}^{2}$. Then $\hat{u}$ is $A$-harmonic in some $\widehat{\Omega} \subset \mathbf{R}^{2}$, for $\widehat{A} \in A_{p}(\lambda, \Lambda)$, in either of the following cases:

$$
\text { (i) } \hat{u}=k u, \quad \text { (ii) } \hat{u}=u(x+z), \quad \text { (iii) } \hat{u}=u(k x) .
$$

Proof. We first note that (i) immediately follows from (iii) in Definition 1.1 and that in this case $\hat{u}$ is $\widehat{A}$-harmonic in $\widehat{\Omega}=\Omega$ with $\widehat{A}=A \in A_{p}(\lambda, \Lambda)$. Similarly, (ii) follows with $\widehat{A}=A(x+z, \xi), \widehat{\Omega}=\Omega-z$ and finally (iii) follows with $\widehat{A}=$ $k^{p-1} A(k x, \xi / k)$ and $\widehat{\Omega}=\Omega / k$.

In the following proofs we will often make the assumptions,

$$
w=0, \quad r=1 \quad \text { and } \max _{\Omega \cap B(w, 2 r)} u=1 .
$$

The assumptions (2.16) are permissible since the parameter $\widehat{M}$ in the definition of a uniform domain is invariant under translations and scalings and that the same is true, see Lemma 2.B, for the constants $\lambda$ and $\Lambda$ used in the definition of $A$-harmonic functions. In addition we will also frequently make use of a test function $\theta$ which satisfies the following,

$$
\theta \in C_{0}^{\infty}(B(0,2)), \quad \theta \equiv 1 \text { on } \bar{B}(0,1), \quad \theta \geq 0 \quad \text { and } \quad|\nabla \theta| \leq c .
$$

After these preliminaries we state, and in some cases also prove, a number of basic lemmas for $A$-harmonic functions.

Lemma 2.1. Let $A \in A_{p}(\lambda, \Lambda)$ for some $(\lambda, \Lambda)$ and suppose that $u$ and $v$ are $A$-harmonic functions in an open set $\Omega$. If

$$
\lim _{y \rightarrow x} \sup v(y) \leq \lim _{y \rightarrow x} \inf u(y)
$$

for all $x \in \partial \Omega$, and also for $x=\infty$ if $\Omega$ is unbounded, and if both sides of the above inequality are not simultaneously $\infty$ or $-\infty$, then $v \leq u$ in $\Omega$

Proof. See [HKM, Theorem 7.6].

Lemma 2.2. Let $\Omega \subset \mathbf{R}^{2}, p \in(1, \infty)$ be given and assume that $A \in A_{p}(\lambda, \Lambda)$ for some $(\lambda, \Lambda)$. Let $w \in \partial \Omega, 0<r \leq r_{0}$ and assume that either $B(w, 2 r) \subset \Omega$ or $w \in \partial \Omega$. Suppose that $u$ is a nonnegative A-harmonic function in $\Omega \cap B(w, 2 r)$ and 
that $u=0$, in the Sobolev sense, on $\Delta(w, 2 r)$ whenever this set is non empty. Then there exists a constant $c_{1} \in[1, \infty)$, depending only on $\Lambda / \lambda$, such that

$$
r^{p-2} \int_{\Omega \cap B(w, r)}|\nabla u|^{p} d x \leq c_{1}^{p}\left(\max _{\Omega \cap B(w, 2 r)} u\right)^{p} .
$$

Proof. We start by assuming (2.16). If $w \in \partial \Omega$ then we extend $u$ to $B(0,2)$, by defining $u \equiv 0$ on $B(0,2) \backslash \Omega$. Then, $u$ is a nonnegative subsolution to (1.5) in $B(0,2)$. In particular, if $\psi \in C_{0}^{\infty}(B(0,2)), \psi \geq 0$, then

$$
\int_{\mathbf{R}^{2}}\langle A(x, \nabla u), \nabla \psi\rangle d x \leq 0 .
$$

To prove that $u$ satisfies $(2.18)$, let $\psi \in C_{0}^{\infty}(B(0,2))$ and put $\tilde{\psi}=[(\eta+\max [u-$ $\left.\epsilon, 0])^{\epsilon}-\eta^{\epsilon}\right] \psi$, with $\epsilon, \eta>0$ small, then $\tilde{\psi}$ is an admissible test function for (1.5). Moreover, using (1.5) we see that

$$
\begin{aligned}
& \int_{u \geq \epsilon}\left[(\eta+\max [u-\epsilon, 0])^{\epsilon}-\eta^{\epsilon}\right]\langle A(x, \nabla u), \nabla \psi\rangle d x \\
& +\int_{u \geq \epsilon}\langle A(x, \nabla u), \nabla u\rangle \epsilon(\eta+u)^{\epsilon-1} \psi d x=0 .
\end{aligned}
$$

From (ii) in Definition 1.1 we note that $\langle A(x, \nabla u), \nabla u\rangle \geq 0$, hence

$$
\int_{u \geq \epsilon}\left[(\eta+\max [u-\epsilon, 0])^{\epsilon}-\eta^{\epsilon}\right]\langle A(x, \nabla u), \nabla \psi\rangle d x \leq 0 .
$$

Using dominated convergence, first letting $\eta \rightarrow 0$ and then $\epsilon \rightarrow 0$ we see that $u$ satisfies (2.18) whenever $\psi \in C_{0}^{\infty}(B(0,2))$ and $\psi \geq 0$. Now, let $\theta$ be as in (2.17) and define $\psi=\theta^{p}(1+u)^{p}$. Then we first see that

$$
\nabla \psi=p \theta^{p-1}(1+u)^{p} \nabla \theta+p \theta^{p}(1+u)^{p-1} \nabla u,
$$

and using (2.21) in (2.18) we can conclude that

$$
p \int_{\mathbf{R}^{2}}\langle A(x, \nabla u), \nabla \theta\rangle \theta^{p-1}(1+u)^{p} d x+p \int_{\mathbf{R}^{2}}\langle A(x, \nabla u), \nabla u\rangle \theta^{p}(1+u)^{p-1} d x \leq 0 .
$$

By using (i) and (ii) in Definition 1.1 we see that

$$
-\Lambda \int_{\mathbf{R}^{2}}|\nabla u|^{p-1}|\nabla \theta| \theta^{p-1}(1+u)^{p} d x+\lambda \int_{\mathbf{R}^{2}}|\nabla u|^{p} \theta^{p}(1+u)^{p-1} d x \leq 0 .
$$

By properties of the test function, we have for a constant $c$ depending only on $\Lambda / \lambda$

$$
\int_{\mathbf{R}^{2}}|\nabla u|^{p} \theta^{p}(1+u)^{p-1} d x \leq c \int_{\mathbf{R}^{2}}|\nabla u|^{p-1} \theta^{p-1}(1+u)^{p} d x,
$$

and by using the normalization of $u$ in (2.16),

$$
\int_{\mathbf{R}^{2}}|\nabla u|^{p} \psi d x \leq c \int_{\mathbf{R}^{2}}|\nabla u|^{p-1} \psi^{(p-1) / p} d x .
$$


By using Hölder's inequality we finally get

$$
\int_{\mathbf{R}^{2}}|\nabla u|^{p} \psi d x \leq c^{p}
$$

for a constant $c$ depending only on $\Lambda / \lambda$, which completes the proof of Lemma 2.1.

Lemma 2.3. Let $p \in(1, \infty)$ be given and assume that $A \in A_{p}(\lambda, \Lambda)$ for some $(\lambda, \Lambda)$. Assume $w \in \mathbf{R}^{2}, 0<r<\infty$ and suppose that $u$ is a positive A-harmonic function in $B(w, 2 r)$. Then there exists a constant $c_{2} \in[1, \infty)$, depending only on $p$ and $\Lambda / \lambda$, such that

$$
\max _{B(w, r)} u \leq c_{2} \min _{B(w, r)} u .
$$

Moreover, if $\hat{p}>2$ and $\hat{p} \leq p<\infty$, then the constant $c_{2}$ can be chosen independent of $p$ but depending on $\hat{p}, \Lambda / \lambda$.

Proof. For a proof of Harnack's inequality in the case $p \in(1, \infty)$ where the constant depends on $p$ we refer the reader to [HKM, Theorem 6.2.]. The proof presented here is based on energy bounds of $\nabla(\log u)$ and works for $p>2$. We note that an alternative proof is given in [KMV]. Assume (2.16) and let $\theta$ be as in (2.17). Let $\epsilon>0$ and define $u_{\epsilon}=(\epsilon+u)$. Using the test function $\theta^{p} u_{\epsilon}^{1-p}$ in (1.5) we see that

$$
0=(1-p) \int_{\mathbf{R}^{2}}\left\langle A(x, \nabla u), \nabla u_{\epsilon}\right\rangle \theta^{p} u_{\epsilon}^{-p} d x+p \int_{\mathbf{R}^{2}}\langle A(x, \nabla u), \nabla \theta\rangle \theta^{p-1} u_{\epsilon}^{1-p} d x
$$

and by properties (i) and (ii) in Definition 1.1 we have,

$$
0 \leq(1-p) \lambda \int_{\mathbf{R}^{2}}|\nabla u|^{p} \theta^{p} u_{\epsilon}^{-p} d x+p c \Lambda \int_{B(0,2)}|\nabla u|^{(p-1)} \theta^{p-1} u_{\epsilon}^{1-p} d x,
$$

and hence

$$
\int_{\mathbf{R}^{2}}|\nabla u|^{p} \theta^{p} u_{\epsilon}^{-p} d x \leq c\left(\frac{p}{p-1}\right) \int_{\mathbf{R}^{2}}|\nabla u|^{(p-1)} \theta^{p-1} u_{\epsilon}^{1-p} d x,
$$

for a constant $c$ depending only on $\Lambda / \lambda$. Therefore, by using Hölders inequality we see that

$$
\int_{\mathbf{R}^{2}}|\nabla u|^{p} \theta^{p} u_{\epsilon}^{-p} d x \leq c^{p}\left(\frac{p}{p-1}\right)^{p} .
$$

Let $\epsilon \rightarrow 0$, then we note that (2.31) in fact states that

$$
\int_{B(0,1)}|\nabla(\log u)|^{p} d x \leq \int_{\mathbf{R}^{2}}|\theta \nabla(\log u)|^{p} d x \leq c^{p}\left(\frac{p}{p-1}\right)^{p} .
$$

Since $f(x)=\log u(x) \in W^{1, p}(B(0,1))$, we may use the Sobolev embedding theorem, which yields, if $p>2$,

$$
\sup _{x, y \in B(0,1)} \frac{|f(x)-f(y)|}{|x-y|^{1-2 / p}} \leq c\left(\int_{B(0,1)}|\nabla f|^{p} d x\right)^{1 / p}
$$


for a constant $\mathrm{c}$ which may depend on $p$. To obtain the uniform in $p$ case, fix $\hat{p}>2$ and let $p>\hat{p}$. Then using (2.33) and Hölder's inequality we see that if $x \in B(0,1)$, then

$$
\begin{aligned}
(|\log u(x)-\log u(0)|)^{\hat{p}} & \leq c^{\hat{p}} \int_{B(0,1)}|\nabla(\log u)|^{\hat{p}} d x \\
& \leq c^{\hat{p}+1-\hat{p} / p}\left(\int_{B(0,1)}|\nabla(\log u)|^{p} d x\right)^{\hat{p} / p} .
\end{aligned}
$$

Based on (2.32) and (2.34) we conclude

$$
|\log u(x)-\log u(0)| \leq c
$$

whenever $x \in B(0,1)$. Hence, $c_{2}^{-1} u(0) \leq u(x) \leq c_{2} u(0)$ whenever $x \in B(0,1)$ for some constant $c$ which is independent of $p$ but depending on $\hat{p}$ and $\Lambda / \lambda$.

Lemma 2.4. Let $\Omega \subset \mathbf{R}^{2}$, assume that $\partial \Omega$ is a Jordan curve and $\Omega$ is a uniform domain with constant $\widehat{M}$. Let $w, r$ and $u$ be as in Lemma 2.2. Then there exist constants $\alpha_{3} \in(0,1]$ and $c_{3}$, both depending only on $\widehat{M}, p, \Lambda / \lambda$, such that if $x, y \in$ $\Omega \cap B(w, r)$ then

$$
|u(x)-u(y)| \leq c_{3}\left(\frac{|x-y|}{r}\right)^{\alpha_{3}} \max _{\Omega \cap B(w, 2 r)} u .
$$

Moreover, if $\hat{p}>2$ and $\hat{p} \leq p<\infty$, then the constants $\alpha_{3}$ and $c_{3}$ can be chosen independent of $p$ but depending on $\widehat{M}, \hat{p}, \Lambda / \lambda$.

Proof. We start with the case $p \in(1, \infty)$ where the constants may depend on $p$. The interior Hölder continuity for A-harmonic functions was proved in [HKM, Theorem 6.6]. If $w \in \partial \Omega$, we note from (ii) in (1.4) and Lemma 2.A that $\lim _{x \rightarrow y} u(x)=0$ for all $y \in \partial \Omega \cap \bar{B}(0, r)$. The result now follows by the same arguments as in [HKM, Theorem 6.44, Lemma 6.47], for constants depending on $p, \widehat{M}$ and $\Lambda / \lambda$. For the uniform in $p$ case, assume (2.16). If $w \in \partial \Omega$ then we extend $u$ to $B(0,2)$ by defining $u \equiv 0$ on $B(0,2) \backslash \bar{\Omega}$. As $u=0$, in the Sobolev sense, on $\Delta(0,2)$ it follows from Lemma 2.2 that $u \in W^{1, p}(B(0,1))$. Hence to prove Lemma 2.4 we can, in both cases, simply use the Sobolev embedding theorem in the form given in (2.33), the Hölder inequality and Lemma 2.2 to obtain

$$
\sup _{x, y \in B(0,1)} \frac{|u(x)-u(y)|}{|x-y|^{1-2 / \hat{p}}} \leq c^{1+1 / \hat{p}-1 / p}\left(\int_{\Omega \cap B(0,1)}|\nabla u|^{p} d x\right)^{1 / p} \leq c
$$

for a constant $c$ which may depend on $\hat{p}$ and $\Lambda / \lambda$ but is independent of $p$. Hence, Lemma 2.4 is valid with $\alpha_{3}=1-2 / \hat{p}$.

Lemma 2.5. Let $\Omega \subset \mathbf{R}^{2}$, assume that $\partial \Omega$ is a Jordan curve and $\Omega$ is a uniform domain with constant $\widehat{M}$. Let $p \in(1, \infty)$, be given and assume that $A \in A_{p}(\lambda, \Lambda)$ for some $(\lambda, \Lambda)$. Let $w \in \partial \Omega, 0<r \leq r_{0}$ and suppose that $u$ is a positive $A$-harmonic 
function in $\Omega \cap B(w, r)$, continuous on $\bar{\Omega} \cap B(w, r)$ with $u=0$ on $\Delta(w, r)$. Then there exists a constant $c_{4}$, depending only on $\widehat{M}, p, \Lambda / \lambda$, such that if $\tilde{r}=r / c_{4}$, then

$$
\max _{\Omega \cap B(w, \tilde{r})} u \leq c_{4} u\left(a_{\tilde{r}}(w)\right)
$$

Moreover, if $\hat{p}>2$ and $\hat{p} \leq p<\infty$, then the constant $c_{4}$ can be chosen independent of $p$ but depending on $\widehat{M}, \hat{p}, \Lambda / \lambda$.

Proof. To prove Lemma 2.5 we proceed as in [CFMS]. We prove the lemma for $\hat{p} \leq p$, but the proof for the case $1<p<\infty$ is similar. Instead of (2.16) we assume $w=0$. Let $k$ be a large number to be chosen later and assume that

$$
k u\left(a_{\tilde{r}}(0)\right)<\max _{\Omega \cap B(0, \tilde{r})} u=u\left(x_{1}\right),
$$

where $x_{1} \in \partial B(0, \tilde{r}) \cap \Omega$ by the maximum principle. We want to derive a contradiction if $k$ is large enough. If $d\left(x_{1}, \partial \Omega\right) \geq \tilde{r} / 100$ then by Harnack's inequality $u\left(x_{1}\right) \leq$ $c u\left(a_{\tilde{r}}(0)\right)$ and hence we obtain a contradiction if $k$ is large enough. Therefore, we assume that $d\left(x_{1}, \partial \Omega\right)<\tilde{r} / 100$. For $c_{4}$ large enough we can connect $x_{1}$ to $a_{\tilde{r}}(0)$ by a Harnack chain totally contained in $B(0,2 r) \cap \Omega$. Thus it follows by Lemma 2.3 that there exist constants $\hat{c}, \lambda \in[1, \infty)$, depending only on $\widehat{M}, \hat{p}$ and $\lambda / \Lambda$, such that

$$
u\left(x_{1}\right) \leq \hat{c}\left(\frac{d\left(a_{\tilde{r}}(0), \partial \Omega\right)}{d\left(x_{1}, \partial \Omega\right)}\right)^{\lambda} u\left(a_{\tilde{r}}(0)\right) .
$$

From (2.39) and (2.40) we see that

$$
\frac{d\left(x_{1}, \partial \Omega\right)}{d\left(a_{\tilde{r}}(0), \partial \Omega\right)} \leq\left(\frac{\hat{c}}{k}\right)^{1 / \lambda}
$$

Let $x_{1}^{+} \in \partial B(0,1) \cap \partial \Omega$ be a point minimizing $\left|x_{1}^{+}-x_{1}\right|$. We apply Lemma 2.4 to $B\left(x_{1}^{+}, \tilde{r} / 2\right)$ to obtain

$$
u\left(x_{1}\right) \leq c_{3} d\left(x_{1}, \partial \Omega\right)^{\alpha_{3}} \max _{\Omega \cap B\left(x_{1}^{+}, \tilde{r} / 2\right)} u
$$

By Harnack's inequality, the maximum principle, our assumption (2.39), (2.41) and (2.42) we obtain, for $x_{2} \in \partial B\left(x_{1}^{+}, \tilde{r} / 2\right) \cap \Omega$,

$$
k \check{c}^{-1} u\left(a_{\tilde{r} / 2}\left(x_{1}^{+}\right)\right) \leq k u\left(a_{\tilde{r}}(0)\right)<u\left(x_{1}\right) \leq c_{3}\left(\frac{\hat{c}}{k}\right)^{\alpha_{3} / \lambda} u\left(x_{2}\right) .
$$

By choosing $k$ so large that $\check{c} c_{3}(\hat{c} / k)^{\left(\alpha_{3} / \lambda\right)}=1$ we obtain,

$$
k u\left(a_{\tilde{r} / 2}\left(x_{1}^{+}\right)\right)<u\left(x_{2}\right) \text { and } u\left(x_{1}\right) \leq u\left(x_{2}\right) .
$$

We can now, thanks to Lemma 2.B, repeat the above argument from (2.39), with (2.43) replacing (2.39), to obtain,

$$
k u\left(a_{\tilde{r} / 2^{m-1}}\left(x_{m-1}^{+}\right)\right)<u\left(x_{m}\right) \text { and } u\left(x_{m}\right) \leq u\left(x_{m+1}\right) .
$$

for every $m \in[1, \infty)$. Since $x_{m} \rightarrow y$ for some $y \in \partial \Omega \cap \bar{B}(0,2 \tilde{r})$ and $u=0$ continuously on $\Delta(0,2 r)$, we conclude that $k u\left(a_{1}(0) \leq 0\right.$ which gives a contradiction. 
Lemma 2.6. Let $\Omega \subset \mathbf{R}^{2}$, assume that $\partial \Omega$ is a Jordan curve and $\Omega$ is a uniform domain with constant $\widehat{M}$. Let $p \in(1, \infty)$, be given and assume that $A \in A_{p}(\lambda, \Lambda)$ for some $(\lambda, \Lambda)$. Assume $w \in \partial \Omega, 0<r \leq r_{0}$ and suppose that $u$ is a positive $A$ harmonic function in $\Omega \cap B(w, r)$, continuous on $\bar{\Omega} \cap B(w, r)$ with $u=0$ on $\Delta(w, r)$. Extend $u$ to $B(w, r)$ by defining $u \equiv 0$ on $B(w, r) \backslash \Omega$. Then there exists a unique finite positive Borel measure $\mu$ on $\mathbf{R}^{2}$ with support in $\Delta(w, r)$, such that whenever $\psi \in C_{0}^{\infty}(B(w, r))$, then

$$
\int_{\mathbf{R}^{2}}\langle A(x, \nabla u), \nabla \psi\rangle d x=-\int_{\mathbf{R}^{2}} \psi d \mu .
$$

Proof. Assume (2.16) and note by (2.18) that if $\psi \in C_{0}^{\infty}(B(0,1))$ and $\psi \geq 0$, then

$$
\int_{\mathbf{R}^{2}}\langle A(x, \nabla u), \nabla \psi\rangle d x \leq 0 .
$$

By using (i) in Definition 1.1, Hölder's inequality and Lemma 2.2, we see that for every compact $K \in B(0,1)$ and every $\psi \in C_{0}^{\infty}(K)$,

$$
\left|\int_{B(0,1)}\langle A(x, \nabla u), \nabla \psi\rangle d x\right| \leq \Lambda \int_{K}|\nabla u|^{p-1}|\nabla \psi| d x \leq c \sup _{K}|\nabla \psi| .
$$

Hence, if $\mu$ is defined according to $(2.44)$, then $\mu$ is a non negative distribution in $B(0,1)$ and hence a positive measure in $B(0,1)$. Since $u$ is $A$-harmonic in $\Omega \cap B(0,1)$ and $u \equiv 0$ in $B(0,1) \backslash \bar{\Omega}, \mu$ has support within $\Delta(0,1)$.

Lemma 2.7. Let $\Omega, w, r, u$ and $\mu$ be as in Lemma 2.6. Then there exists a constant $c_{6}$, depending only on $\widehat{M}, p, \Lambda / \lambda$, such that if $\tilde{r}=r / c_{6}$, then

$$
c_{6}^{-p} \tilde{r}^{p-2} \mu(\Delta(w, \tilde{r})) \leq\left(u\left(a_{\tilde{r}}(w)\right)\right)^{p-1} \leq c_{6}^{p} \tilde{r}^{p-2} \mu(\Delta(w, \tilde{r})) .
$$

Moreover, there exists $\hat{p}>2$ such that if $\hat{p} \leq p<\infty$, then the constant $c_{6}$ can be chosen independent of $p$ but depending on $\widehat{M}, \hat{p}, \Lambda / \lambda$.

Proof. For the case $1<p<\infty$ with a constant depending on $p$ we refere to [KZ]. For the uniform in $p$ case, note that by Lemma 2.3 and Lemma 2.5, there exists a constant $\tilde{c}$, depending only on $\widehat{M}, \hat{p}$ and $\Lambda / \lambda$, such that if $\tilde{r}=r / \tilde{c}$, then

$$
\tilde{c}^{-1} u\left(a_{\tilde{r}}(w)\right) \leq \max _{\Omega \cap B(w, 4 \tilde{r})} u \leq \tilde{c} u\left(a_{\tilde{r}}(w)\right) .
$$

Moreover, instead of (2.16) we assume that

$$
w=0, \quad \tilde{r}=1 \quad \text { and } \quad u\left(a_{\tilde{r}}(w)\right)=1 .
$$

Therefore, to prove Lemma 2.7 it suffices to show the existence of $c_{6}$, depending only on $\widehat{M}, \hat{p}, \Lambda / \lambda$, such that

$$
c_{6}^{-p} \leq \mu(\Delta(0,1)) \leq c_{6}^{p} .
$$


Let $\theta$ be as in (2.17). Using Lemma 2.6, (i) in Definition 1.1, Hölder's inequality, Lemma $2.2,(2.48)$ and the normalization in (2.49), we see that

$$
\begin{aligned}
\mu(\Delta(0,1)) & \leq \int_{\mathbf{R}^{2}} \hat{\theta} d \mu \leq \int_{\mathbf{R}^{2}}|\nabla \hat{\theta}||A(x, \nabla u)| d x \leq \int_{\Omega \cap B(0,2)} c \Lambda|\nabla u|^{p-1} d x \\
& \leq c\left(\int_{\Omega \cap B(0,2)}|\nabla u|^{p} d x\right)^{(p-1) / p} \leq c c_{1}^{p-1} \leq c_{6}^{p} .
\end{aligned}
$$

Hence, the right hand side inequality in (2.50) is proved.

Next we prove the left hand side inequality in (2.50). Our proof is based on [KZ], see also [EL]. We define

$$
M(\rho)=\sup _{B(0, \rho)} u(x) \text { whenever } \rho \in[0,2] .
$$

Let $h$ be $A$-harmonic in $B(0,1)$, to the same $A(x, \eta)$ as $u$, with boundary values equal to $u$ on $\partial B(0,1)$. Note that by assumption $u$ is continuous on $\bar{\Omega} \cap \bar{B}(0,1)$ and hence $u$ is well defined on $\partial B(0,1)$. By the comparison principle for $A$-harmonic functions we see that $0 \leq u \leq h$ in $B(0,1)$. Moreover, considering $p>\hat{p}$ and applying Lemma 2.3 to the function $h$ we see that

$$
\inf _{B(0,1 / 2)} h \geq c_{2}^{-1} \sup _{B(0,1 / 2)} h \geq c_{2}^{-1} \sup _{B(0,1 / 2)} u=c_{2}^{-1} M(1 / 2)
$$

Using Lemma 2.4 we see that

$$
u(x) \leq c_{3} t^{\alpha_{3}} M(1 / 2) \text { whenever } x \in B(0, t), t<1 / 4 .
$$

Let $\beta=1 /\left(2 c_{2}\right)$ and restrict $t$ to the interval $\left[0,\left(\beta / c_{3}\right)^{1 / \alpha_{3}}\right)$. Using $(2.54)$ it then follows that $M(t) \leq \beta M(1)$. Under the same conditions we also see, using (2.53), that, whenever $x \in B(0, t)$,

$$
h(x)-u(x) \geq \inf _{B(0,1 / 2)} h-\sup _{B(0, t)} u \geq 2 \beta M(1 / 2)-\beta M(1 / 2)=\beta M(1 / 2) .
$$

Next we note that the function

$$
\psi=\min _{B(0,1)}\{h-u, \beta M(1 / 2)\}
$$

is non negative in $B(0,1)$ and belongs to the space $W_{0}^{1, p}(B(0,1))$. Using $(2.55)$ we also see that $\psi=\beta M(1 / 2)$ on $B(0, t)$. Now, we observe that

$$
\int_{B(0,1)}|\nabla \psi|^{p} d x \leq \int_{B(0,1)}(|\nabla h|+|\nabla u|)^{p-2}|\nabla h-\nabla u|^{2} d x .
$$


By using (ii) in Definition 1.1, that $h$ is A-harmonic in $B(0,1)$ and Lemma 2.6 we also see that

$$
\begin{aligned}
& \lambda \int_{B(0,1)}(|\nabla h|+|\nabla u|)^{p-2}|\nabla h-\nabla u|^{2} d x \\
& \leq \int_{B(0,1)}\langle A(x, \nabla h)-A(x, \nabla u), \nabla \psi\rangle d x \\
& =-\int_{B(0,1)}\langle A(x, \nabla u), \nabla \psi\rangle d x=\int_{B(0,1)} \psi d \mu,
\end{aligned}
$$

based on which we can conclude that

$$
\int_{B(0,1)}|\nabla \psi|^{p} d x \leq \frac{\beta}{\lambda} M(1) \mu(B(0,1)) .
$$

But on the other hand using Hölder's inequality as well as a Sobolev type inequality we see that

$$
(\beta M(1))^{p} \pi t^{2} \leq \int_{B(0,1)}|\psi|^{p} d x \leq c^{p} \int_{B(0,1)}|\nabla \psi|^{p} d x .
$$

An explicit constant, independent of $p$, for which the right hand inequality in (2.60) is true can be found in [GT]. Combining (2.59) and (2.60) we can therefore conclude that

$$
(\beta M(1 / 2))^{p} \pi t^{2} \leq c^{p} \beta M(1 / 2) \mu(B(0,1))
$$

for a constant $c$ independent of $p$. Hence, by the assumption (2.49) and if we choose $t=\left(\beta / c_{3}\right)^{1 / \alpha_{3}} / 2$, then

$$
c_{6}^{-p} \leq \mu(\Delta(0,1)) .
$$

This completes the proof of the left hand side inequality in (2.50) and hence the proof of Lemma 2.7.

Remark. Lemmas 2A, 2B and Lemmas $2.2-2.7$ are valid in $\mathbf{R}^{n}, n \geq 3$ as well if we assume that $\Omega$ is a NTA-domain. In particular, the restriction to $\mathbf{R}^{2}$ is only necessary in the proof of Theorem 1.3 and Theorem 1.4 below and not in this section.

\section{Proof of the main results}

Proof of Theorem 1.3 and Theorem 1.4. The proof of Theorem 1.3 and Theorem 1.4 are identical apart from the constants and we therefore only prove Theorem 1.4. Theorem 1.3 then follows. The proof is based on the proof of Lemma 2.16 in [BL]. In fact to prove Theorem 1.4 we repeat the argument in [BL, Lemma 2.16] making sure that the constants of our estimates can be chosen independently of $p$ whenever $p \geq \hat{p}>2$. We note that the following proof is identical with the proof of Theorem 2 in [LN2], but for the readers convenience, we include the proof. In the following we let $\hat{p}$ be large enough to ensure the validity of the statements in Lemmas 2.1-2.4 and in Lemma 2.6. Moreover, we let $\tilde{r}=r /\left(100 c_{6}\right)$ with $c_{6}$ as in Lemma 2.6.

Let $\gamma:[0,1] \rightarrow \mathbf{R}^{2}$ be a parametrization of $\partial \Omega$ such that $\gamma(0)=w$. Let $r_{1}=\tilde{r} / \tilde{c}$ where $\tilde{c}=\tilde{c}(\widehat{M}) \geq 1$ will be chosen later. Let $t_{1}=\sup \left\{t<0:|\gamma(t)-w|=r_{1}\right\}, t_{2}=$ 
$\inf \left\{t<0:|\gamma(t)-w|=r_{1}\right\}, z_{1}=\gamma\left(t_{1}\right)$ and $z_{2}=\gamma\left(t_{2}\right)$. Then $\left|w-z_{1}\right|=\left|w-z_{2}\right|=r_{1}$ and the part of $\partial \Omega$ between $z_{1}$ and $z_{2}$ is contained in $B\left(w, r_{1}\right)$, see the Figure 1 . If $r_{2}=r_{1} / \tilde{c}$, then from (1.2) we see, for $\tilde{c}$ large enough, that $B\left(z_{1}, r_{2}\right) \cap B\left(z_{2}, r_{2}\right)=\emptyset$. For any two points $\zeta_{1} \in \Delta\left(z_{1}, r_{2}\right)$ and $\zeta_{2} \in \Delta\left(z_{2}, r_{2}\right)$ we can use (1.3) to construct a curve with endpoints $\zeta_{1}, \zeta_{2}$ in the following way. Take $\rho$ such that $B\left(\zeta_{i}, \rho\right) \subset B\left(z_{i}, r_{2}\right)$ for $i=1,2$. Draw the curve from $a_{\rho}\left(\zeta_{1}\right)$ to $a_{\rho}\left(\zeta_{2}\right)$ guaranteed by (1.3). Similarly, connect $a_{\rho}\left(\zeta_{1}\right)$ to $a_{\rho / 2}\left(\zeta_{1}\right)$ and then $a_{\rho / 2}\left(\zeta_{1}\right)$ to $a_{\rho / 4}\left(\zeta_{1}\right)$ and so on. Since $a_{\rho / 2^{n}}\left(\zeta_{1}\right) \rightarrow \zeta_{1}$, as $n \rightarrow \infty$, this curve ends up at $\zeta_{1}$. We can advance from $a_{\rho}\left(\zeta_{2}\right)$ to $\zeta_{2}$ in the same way. The total curve from $\zeta_{1}$ to $\zeta_{2}$ is denoted by $\Gamma$, see Figure 1.

From our construction and (1.3) we note that, for $\tilde{c}$ large enough,

(i) $\Gamma \backslash\left\{\zeta_{1}, \zeta_{2}\right\} \subset \Omega \cap B(w, \tilde{r})$,

(ii) $H^{1}(\Gamma) \leq \tilde{c} \tilde{r}$

(iii) $\min \left\{H^{1}(\Gamma([0, t])), H^{1}(\Gamma([t, 1]))\right\} \leq \tilde{c} d(\Gamma(t), \partial \Omega)$.

Recall that $H^{1}(\cdot)$ denotes the one-dimensional Hausdorff measure on $\Gamma$. In the following we let $\tilde{c}$ be large enough to satisfy the above requirements and to make sure that we can connect any to points in $B(w, \tilde{r})$ by a Harnack chain totally contained in $B(0, r) \cap \Omega$.

Next we consider the functions $u, v$ in Theorem 1.4, we extend both of these to $B(w, r)$ in the standard way and we assume, as we may, that $u\left(a_{\tilde{r}}(w)\right)=v\left(a_{\tilde{r}}(w)\right)=$ 1. We let $\mu$ and $\nu$ be the measures, in the sense of Lemma 2.5, corresponding to $u$ and $v$ respectively. Let $M_{+}=M_{+}(\widehat{M})$ be a constant to be chosen and assume that $M_{+}$is so large that $\Gamma \cap B\left(w, r_{1} / M_{+}\right)=\emptyset$ independently of $\zeta_{1} \in \Delta\left(z_{1}, r_{2}\right)$ and $\zeta_{2} \in \Delta\left(z_{2}, r_{2}\right)$. Existence of $M_{+}$follows from (3.63).

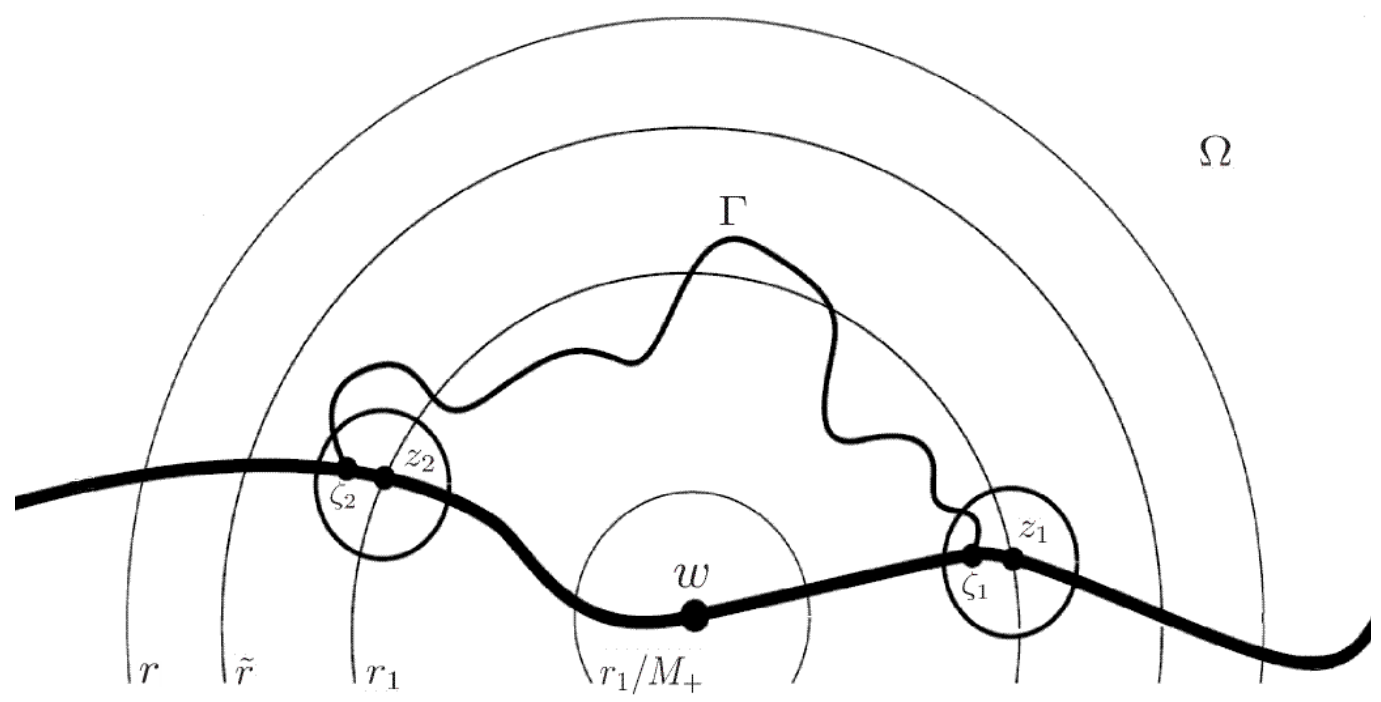

Figure 1. The curve $\Gamma$.

Suppose that $u / v>\lambda$ at some point in $\Omega \cap B\left(w, r_{1} / M_{+}\right)$. Our intention is to prove that $\lambda$ can not be too large. Using the continuity of $u$ and $v$ in $\bar{\Omega} \cap B(w, r)$, the comparison principle for $A$-harmonic functions and that $\Omega \subset \mathbf{R}^{2}$, we see that $u / v>\lambda$ at some point $\xi \in \Gamma$. Making use of the point $\xi$ and using (3.63), Lemma 2.6 
and Lemma 2.2 we deduce for some $s, 0<s<\tilde{r} / 2$ and $i \in\{1,2\}$, that

$$
\frac{\mu\left(\Delta\left(\zeta_{i}, s\right)\right)}{\nu\left(\Delta\left(\zeta_{i}, s\right)\right)} \geq \frac{c_{6}^{-p}\left(u\left(a_{s}\left(\zeta_{i}\right)\right)\right)^{p-1}}{c_{6}^{p}\left(v\left(a_{s}\left(\zeta_{i}\right)\right)\right)^{p-1}}>\check{c}^{-p} \lambda^{p-1},
$$

where $\check{c}$ is a constant independent of $p$. Allowing $\zeta_{i}$ in this construction to vary in $\Delta\left(z_{i}, r_{2}\right), i=1,2$, we get a covering of either $\Delta\left(z_{1}, r_{2}\right)$ or $\Delta\left(z_{2}, r_{2}\right)$ by balls of the form $\Delta_{\zeta}=\Delta(\zeta, s)$. Assume for example that $\Delta\left(z_{1}, r_{2}\right)$ is covered by balls of this type. Then using a standard covering argument we get a subcovering, $\left\{\Delta_{\zeta_{n}}\right\}$, of $\Delta\left(z_{1}, r_{2}\right)$ such that the balls, with one-fifth the diameter of the original balls but the same centers, denoted $\left\{\Delta_{\zeta_{n}}^{*}\right\}$, are disjoint. From (3.64), Lemmas 2.2 and Lemma 2.6 we then deduce

$$
\check{c}^{-p} \lambda^{p-1} \nu\left(\Delta\left(z_{1}, r_{2}\right)\right) \leq \check{c}^{-p} \lambda^{p-1} \nu\left(\cup_{n} \Delta_{\zeta_{n}}\right)<\sum \mu\left(\Delta_{\zeta_{n}}\right) \leq \hat{c}^{p} \sum \mu\left(\Delta_{\zeta_{n}}^{*}\right)
$$

for yet another constant $\hat{c}_{6}$ which is independent of $p$. Hence

$$
\check{c}^{-p} \lambda^{p-1} \nu\left(\Delta\left(z_{1}, r_{2}\right)\right)<\hat{c}^{p} \mu(\Delta(w, 2 \tilde{r})) .
$$

Moreover, using Lemma 2.6 and Lemma 2.2 once more we also see that

$$
\nu\left(\Delta(w, 2 \tilde{r}) \leq c^{p} \nu\left(\Delta\left(z_{1}, r_{2}\right)\right)\right.
$$

for some $c$ independent of $p$. Combining (3.66) and (3.67) we first see that

$$
c^{-p} \check{c}^{-p} \hat{c}^{-p} \lambda^{p-1}<\frac{\mu(\Delta(w, 2 \tilde{r}))}{\nu(\Delta(w, 2 \tilde{r}))}
$$

and then, using Lemma 2.6, Lemma 2.2 and the normalization $u\left(a_{\tilde{r}}(w)\right)=1=$ $v\left(a_{\tilde{r}}(w)\right)$,

$$
c^{-p} \check{c}^{-p} \hat{c}^{-p} \lambda^{p-1}<\frac{\mu(\Delta(w, 2 \tilde{r}))}{\nu(\Delta(w, 2 \tilde{r}))} \leq \bar{c}^{p}
$$

for yet another constant $\bar{c}$ which is independent of $p$. (3.69) implies that $\lambda<c$ with $c$ independent of $p$. Hence $u / v \leq c$ in $\Omega \cap B\left(w, \tilde{r} / M_{+}\right)$and the proof of Theorem 1.4 is complete.

Proof of Theorem 1.7. Let $u$ and $v$ be as in the statement of Theorem 1.7 and note that this implies, in particular, that $u, v \in W^{1, \infty}(\Omega \cap B(w, r / 2)) \cap C(\bar{\Omega} \cap$ $\bar{B}(w, r / 2))$. We let, for $p \in(2, \infty), u_{p}$ and $v_{p}$ be the unique weak solutions to the problem (1.15) in $\Omega \cap B(w, r / 2)$, continuous on $\bar{\Omega} \cap B(w, r / 2)$ with $u_{p}=0=v_{p}$ on $\Delta(w, r / 2)$ and $\lim _{x \rightarrow y} u_{p}(x)=u(y), \lim _{x \rightarrow y} v_{p}(x)=v(y)$ for all $y \in \partial B(w, r / 2) \cap$ $\Omega$. Existence of $u_{p}$ and $v_{p}$ follows from Lemma 2.A. Using the uniqueness result of Juutinen [Ju, Theorem 4.25] together with the result concerning the limit [Ju, Theorem 1.15, Proposition 2.5 and Corollary 3.8] we see that there exists a sequence $\left\{p_{j}\right\}, p_{j} \rightarrow \infty$ as $j \rightarrow \infty$, such that $u_{p_{j}} \rightarrow u$ and $v_{p_{j}} \rightarrow v$ uniformly in $\bar{\Omega} \cap B(w, r / 2)$ as $j \rightarrow \infty$. In particular, the unique solutions $u$ and $v$ to the Dirichlet problem in (1.11) is the uniform limit as $p_{j} \rightarrow \infty$, of the corresponding unique solutions $u_{p}$ and $v_{p}$ to the problem (1.15). It follows that for every $\epsilon>0$ there exists $\tilde{p}=\tilde{p}(\epsilon)>2$ such that if $p>\tilde{p}$ then

$$
\left|u_{p}(x)-u(x)\right|<\epsilon \quad \text { and } \quad\left|v_{p}(x)-v(x)\right|<\epsilon
$$


whenever $x \in \bar{\Omega} \cap \bar{B}(w, r / 2)$. Let $\hat{p}$ and $c$ be as in Theorem 1.4. Then for $p \geq \hat{p}$ and $c_{3}=2 c_{2}$

$$
c_{3}^{-1} \frac{u_{p}\left(a_{r}(w)\right)}{v_{p}\left(a_{r}(w)\right)} \leq \frac{u_{p}(x)}{v_{p}(x)} \leq \hat{c}_{3} \frac{u_{p}\left(a_{r}(w)\right)}{v_{p}\left(a_{r}(w)\right)} \text { whenever } x \in \Omega \cap B\left(w, r / \hat{c}_{3}\right) .
$$

Since $c_{3}$ is independent of $p$, theorem 1.7 follows from (3.70) and (3.71).

\section{References}

[A1] Aronsson, G.: Minimization problems for the functional $\sup _{x} F\left(x, f(x), f^{\prime}(x)\right)$. - Ark. Mat. 6, 1965, 33-53.

[A2] Aronsson, G.: Minimization problems for the functional $\sup _{x} F\left(x, f(x), f^{\prime}(x)\right)$ II. - Ark. Mat. 6, 1966, 409-431.

[A3] Aronsson, G.: Extension of functions satisfying Lipschitz conditions. - Ark. Mat. 6, 1967, $551-561$.

[A4] Aronsson, G.: On the partial differential equation $u_{x}^{2} u_{x x}+2 u_{x} u_{y} u_{x y}+u_{y}^{2} u_{y y}$. Ark. Mat. 7, 1968, 395-425.

[ALuN] Avelin, B., N. Lundström, and K. Nyström: Boundary estimates for solutions to operators of $p$-Laplace type with lower order terms. - Submitted.

[BEJ] Barron, E., C. Evans, and R. Jensen: The infinity Laplacian, Aronsson's equations and their generalizations. - Trans. Amer. Math. Soc. 360, 2008, 77-101.

[BL] Bennewitz, B., and J. Lewis: On the dimension of $p$ harmonic measure. - Ann. Acad. Sci. Fenn. 30, 2005, 459-505.

[CFMS] Caffarelli, L., E. Fabes, S. Mortola, and S. Salsa: Boundary behavior of nonnegative solutions of elliptic operators in divergence form. - Indiana J. Math. 30:4, 1981, 621-640.

[EL] ERemenko, A., and J. Lewis: Uniform limits of certain $A$-harmonic functions with applications to quasiregular mappings. - Ann. Acad. Sci. Fenn. Math. 16, 1991, 361-375.

[GZ] Gariepy, R., and W. P. Ziemer: A regularity condition at the boundary for solutions of quasilinear elliptic equations. - Arch. Ration. Mech. Anal. 67, 1977, 25-39.

[G] Gehring, F.: Uniform domains and the ubiquitous quasidisk. - Jahresber. Deutsch. Math.Verein. 89:2, 1987, 88-103.

[GT] GILBARG, D., and N. S. TRUdinger: Elliptic partial differential equations of second order. - Reprint of the 1998 edition, Springer-Verlag, 2001.

[HKM] Heinonen, J., T. Kilpeläinen, and O. Martio: Nonlinear potential theory of degenerate elliptic equations. - Oxford Univ. Press, Oxford, 1993.

[J] Jensen, R.: Uniqueness of Lipschitz extensions minimizing the sup-norm of the gradient. - Arch. Ration. Mech. Anal. 123, 1993, 51-74.

[JWY] Jensen, R., C. WANG, and Y. YU: Uniqueness and nonuniqueness of viscosity solutions to Aronsson's equation. - Arch. Ration. Mech. Anal. 190, 2008, 347-370.

[JK] JeRISOn, D., and C. Kenig: Boundary behaviour of harmonic functions in nontangentially accessible domains. - Adv. Math. 46, 1982, 80-147.

[Ju] JuUtinen, P.: Minimization problems for Lipschitz functions via viscosity solutions. Ann. Acad. Sci. Fenn. Math. Diss. 115, 1998, 1-53.

[KZ] Kilpeläinen, T., and X. Zhong: Growth of entire $A$-subharmonic functions. - Ann. Acad. Sci. Fenn. Math. 28, 2003, 181-192.

[KMV] Koskela, P., J. J. Manfredi, and E. Villamor: Regularity theory and traces of $A$ harmonic functions. - Trans. Amer. Math. Soc. 348:2, 1996. 
[LLuN] Lewis, J., N. Lundström, and K. Nyström: Boundary Harnack inequalities for operators of $p$-Laplace type in Reifenberg flat domains. - In: Perspectives in PDE, Harmonic Analysis and Applications, Proceedings of Symposia in Pure Mathematics 79, 2008, 229266.

[LN1] Lewis, J., and K. Nyström: Boundary behavior for p-harmonic functions in Lipschitz and starlike Lipschitz ring domains. - Ann. Sci. École Norm. Sup. (4) 40:4, 2007, 765-813.

[LN2] Lewis, J., and K. Nyström: The boundary Harnack inequality for infinity harmonic functions in the plane. - Proc. Amer. Math. Soc. 136, 2008, 1311-1323.

[LN3] Lewis, J., and K. Nyström: Boundary behaviour of $p$-harmonic functions in domains beyond Lipschitz domains. - Adv. Calc. Var. 1, 2008, 133-170.

[LN4] Lewis, J., and K. Nyström: Boundary behaviour and the Martin boundary problem for p-harmonic functions in Lipschitz domains. - Ann. of Math. (2) (to appear).

Received 30 April 2010 